Anne-Cécile Lamy-Joswiak

DOI: $10.4312 /$ vestnik.13.597-598

Faculté des lettres, Université de Ljubljana

Slovénie

annececile.lamyjoswiak@ff.uni-lj.si

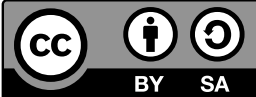

\title{
FORMATION HYBRIDE EN LANGUES : ARTICULER PRÉSENTIEL ET DISTANCIEL
}

Nissen Elke (2019). Formation hybride en langues : articuler présentiel et distanciel. Paris : Les Éditions Didier. Collection Langues et didactique. ISBN : 978-2-278-09400-4. Broché, 284 pages. $19 €$

Elke Nissen est germaniste, professeure en didactique des langues et ingénierie éducative numérique à l'Université Grenoble Alpes et membre permanente du LIDILEM, Laboratoire de linguistique et didactique des langues étrangères et maternelles, au sein duquel elle mène des recherches sur les formations hybrides en langues (FHL), en collaboration avec des chercheurs du monde entier.

Dans son ouvrage intitulé Formation hybride en langues : articuler présentiel et distanciel, elle s'appuie sur un certain nombre de recherches et de projets d'ingénierie pédagogique menés ces quinze dernières années dans le domaine des dispositifs médiatisés appliqués à l'enseignement-apprentissage des langues propre à l'enseignement supérieur, pour analyser le fonctionnement et les spécificités des FHL et ainsi, mieux comprendre « comment elles sont conçues, perçues et vécues, les relations qui s'y créent, les interactions qui s'y déroulent, etc. » (p.11).

Découpé en 6 chapitres, l'ouvrage propose une étude complète des travaux de recherche sur les FHL en décrivant précisément la mise en place de ce type de formation dans des contextes universitaires différents. Tout d'abord, l'auteure examine les apports de la « coprésence des modes présentiel et distanciel » (p.35) et les modalités synchrone/ asynchrone qui permettent de définir et délimiter le concept de FHL (chapitre 1). Elle souligne l'importance des scénarios de communication qui serviront de « fil rouge ou fil conducteur » (p.73) à l'élaboration d'une telle formation et rappelle la nécessité de contextualiser la FHL en fonction des besoins des apprenants (chapitre 2). Elle se focalise sur l'un des paramètres qui interviennent dans l'articulation des modes synchrone/ asynchrone, à savoir l'approche par tâche(s), qui permet de scénariser la formation et de la rendre cohérente (chapitre 3). Ensuite, l'auteure présente trois projets pédagogiques, Cultura, English language et Galanet, qui combinent télécollaboration et présentiel afin 
d'accentuer la dimension interculturelle de l'enseignement-apprentissage d'une langue étrangère (chapitre 4). Ces FHL télécollaboratives représentent un «type d'authenticité particulier » puisque les apprenants sont placés dans une relation contrastive et conscientisée entre la ou les culture(s) cible(s) et la ou les cultures d'origine (p.189). Enfin, l'auteure insiste sur l'importance « d'accompagner l'apprenant dans une FHL » (p.201) en développant sa compétence d'autonomie et en soutenant son autonomisation grâce au tuteur, à la dynamique de groupe et au travail collectif à distance (chapitre 5). Pour cela, la planification et l'instauration de " contraintes » (comme le dépôt de travaux sur une plateforme) et d'une « cadence régulière» (p.214) s'imposent. Ce type de formations pouvant être chronophage tant pour l'enseignant que pour l'étudiant, l'auteure rappelle dans sa conclusion qu'il est capital de veiller à la gestion du temps (chapitre 6).

La méthodologie de l'auteure est rigoureuse et basée sur une littérature scientifique abondante. De nombreuses notions clés jalonnent ce panorama des recherches en FHL pour mieux en éclairer les problématiques : le tandem présentiel/distanciel, la tâche, la perspective actionnelle, l'authenticité (de la tâche), l'autonomie (des apprenants), la compétence interculturelle, le tutorat, la dynamique de groupe, etc. Chaque FHL est illustrée par des schémas qui permettent de saisir les conditions concrètes de sa réalisation et expliquent les modalités d'articulation entre le présentiel et le distanciel au niveau des scénarios communicatifs et pédagogiques. Enfin, des tableaux synthétiques et des reprises présentes au sein de chaque chapitre facilitent grandement la lecture de l'ouvrage.

Ainsi Elke Nissen offre-t-elle une synthèse dense et minutieuse des travaux de recherche et des projets d'ingénierie menés dans l'enseignement supérieur depuis le début des années 2010. La taxonomie et la typologie des FHL qu'elle livre en guise de conclusion constituent désormais un outil essentiel aux (futurs) enseignants et concepteurs de formation qui trouveront dans cet ouvrage de référence un soutien indéniable à la mise en place ou la réévaluation d'une FHL, alors que la littérature francophone sur le numérique en didactique des langues s'amplifie. À ce titre, l'auteure propose une réflexion rigoureuse et résolument didactique sur les FHL, où théorie et pratique s' articulent brillamment, c'est pourquoi son ouvrage s'adresse aussi bien aux enseignants, aux chercheurs qu'aux ingénieurs pédagogiques, auxquels l'enseignement à distance et/ou hybride s'impose dans ce contexte de crise sanitaire. 